ISSN: 0210-1696

DOI: http://dx.doi.org/10.14201/scero20154642544

\title{
EL SÍNDROME DEL CROMOSOMA X FRÁGIL: FENOTIPO CONDUCTUAL Y DIFICULTADES DE APRENDIZAJE ${ }^{1}$
}

\section{The Fragile X Syndrome: Behavioral Phenotype and Learning Disabilities}

Claudia Grau Rubio

Departamento de Didáctica y Organización Escolar. Facultad de Magisterio. Universidad de Valencia. Av. Tarongers, 4. 46022 Valencia

Claudia.grau@uv.es

María Fernández HawrylaK

Departamento de Ciencias de la Educación. Facultad de Humanidades y Educación. Universidad de Burgos

José Luis Cuesta Gómez

Departamento de Ciencias de la Educación. Facultad de Humanidades y Educación. Universidad de Burgos

Recepción: 7 de mayo de 2015

Fecha de aceptación definitiva: 29 de septiembre de 2015

Biblid. [0210-1696 (2015) vol. 46 (4), n. ${ }^{\circ} 256$, octubre-diciembre; 25-44]

Resumen: En este artículo se describe el fenotipo conductual de las personas con el Síndrome X Frágil y las repercusiones que tiene en el ámbito educativo. Este síndrome se caracteriza por problemas de integración sensorial, déficits cognitivos (razonamiento verbal, habilidades abstractas/visuales y cuantitativas, memoria a corto plazo, procesamiento secuencial, atención y procesos ejecutivos), trastornos del lenguaje (fonéticofonológicos, semánticos, morfosintácticos y pragmáticos) y de la comunicación, ansiedad social, hiperexcitación general, autismo, dificultades sociales no autistas, déficit de atención e hiperactividad, y dificultades de aprendizaje. El fenotipo conductual es muy variable y depende del sexo, de la edad y de si estas personas presentan mutación completa o premutación. El fenotipo conductual tiene repercusiones importantes en el

1 Los autores son miembros del Instituto de Investigación Polibienestar de la Universidad de Valencia. 
ámbito de la educación, ya que nos permite entender las dificultades de aprendizaje y conductuales de las personas con Síndrome del X Frágil, y desarrollar estrategias de intervención específicas.

Palabras clave: Síndrome X Frágil; fenotipo conductual; perfil cognitivo; perfil psicopatológico; lenguaje.

Aвstract: In this article, we describe the behavioral phenotype of individuals with Fragile X Syndrome and its impact in the educational scope. This syndrome is characterized by difficulties in sensory integration, cognitive deficits (verbal reasoning, abstract/ visual and cuantitative skills, short term memory, sequential processing, attention and executive processes), language disorders (phonetic-phonologicals, semanticals, morphosyntacticals and pragmaticals) and communication disorders, social anxiety, general hyperarousal, autism, non autistic social difficulties, attention deficit and hyperactivity, and learning disabilities. The behavioral phenotype is highly variable and depends on sex, age, and mutation status (full mutation or premutation). The behavioural phenotype has important repercussions in education, as it enables us to understand the learning disabilities and to develop specific intervention strategies.

KeYs words: Fragile X Syndrome; neurobehavioral manifestations; cognitive symptoms; psychopathology; language.

\section{Introducción}

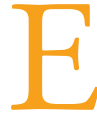

L FENOTIPO CONDUCTUAL ES UN PATRÓN CARACTERÍSTICO de observaciones motoras, cognitivas, lingüísticas y sociales que se asocian de forma consistente con un trastorno biológico/genético (Artigas-Pallarés et al., 2006; O’Brien, 2006), e implica que las personas afectadas por un síndrome genético tienen más probabilidades de mostrar dichas características y comportamientos atípicos (Di Nuovo y Buono, 2011). Este patrón tiene una base biológica, sin perjuicio de que lo ambiental pueda tener influencia en su desarrollo (Ruggieri y Arberas, 2003). No obstante, todas las personas con un síndrome no muestran todas las conductas características de éste y tampoco lo harán en el mismo grado o etapa evolutiva (Hopdapp y Dykens, 2004; Hopdapp y Dykens, 2005).

El Síndrome del cromosoma X Frágil (en adelante SXF) es la causa más frecuente de discapacidad intelectual de origen hereditario y la segunda cromosopatía después del Síndrome de Down (en adelante SD). Está provocado por una mutación del gen FMR1, localizado en un extremo del brazo largo del cromosoma Xq27.3. Esta mutación consiste en una expansión del gen FMR1 de 200 o más repeticiones del trinucleótido CGG (Citosina, Guanina, Guanina), lo que origina un déficit en la producción de la proteína FMRP (García-Nonell et al., 2006).

En la población en general, el número de repeticiones CGG oscila entre 5 y 50 ; el aumento del número de repeticiones entre 50 y 200 corresponde al estado de premutación; cuando el número de repeticiones es superior a 200, la situación es de mutación 
completa. La mutación completa lleva consigo un estado de hipermetilación que va a inhibir la expresión del gen FMR1 y, por tanto, la síntesis de la proteína FMRP, responsable de las manifestaciones clínicas del Síndrome (Fernández-Lozano et al., 2011). La reducción o falta de FMRP tiene un efecto negativo en el desarrollo y funcionamiento del cerebro y en la correcta formación de las sinapsis (maduración, plasticidad, guía axonal y poda sináptica), que son la causa de los trastornos cognitivos, emocionales y comportamentales (Reiss y Dant, 2003; Abbeduto et al., 2007; Di Nuovo y Buono, 2011).

El SXF afecta a ambos sexos, pero con mayor frecuencia a los varones que a las mujeres, ya que éstas pueden compensar en parte el defecto ocasionado por el cromosoma X mutado con el otro cromosoma X. Esta compensación no se puede llevar a cabo en el varón, al disponer de un único cromosoma X (Artigas-Pallarés et al., 2001).

En el SXF, el fenotipo conductual varía en función del sexo, de la edad y de si los afectados presentan mutación completa o premutación, y tiene repercusiones importantes para la intervención psicoeducativa y clínica (Cornish et al., 2008).

El objetivo de este artículo es describir el fenotipo conductual del SXF y sus repercusiones en la educación. Así, el fenotipo conductual permite a los profesionales de la educación conocer cómo procesan la información, cómo es el lenguaje, cuáles son las características comportamentales de las personas con $\mathrm{SXF}$, en función de su edad, sexo y de si presentan mutación o premutación; también, determinar cuáles son las fortalezas y debilidades del alumnado con SXF, la naturaleza de las dificultades de aprendizaje y de sus conductas desadaptativas, diseñar las estrategias de intervención educativa adecuadas, organizar el entorno educativo y el aula, manejar los comportamientos desadaptativos, y desarrollar estrategias para facilitarles el aprendizaje.

\section{Fenotipo conductual y etapas evolutivas}

Las manifestaciones del Síndrome son diferentes según las etapas evolutivas. Tanto los niños como las niñas y los adolescentes con SXF con mutación completa tienen un alto riesgo de tener un perfil característico de problemas comportamentales, cognitivos y emocionales desde el comienzo de la infancia que es cualitativamente similar, pero cuantitativamente diferente en niños y en niñas. Por el contrario, los sujetos con premutación muestran un desarrollo normal, a no ser que la longitud de las repeticiones CGG sean mayores de 100. En este supuesto, las manifestaciones tienen una gran variabilidad (Reiss y Dant, 2003).

Los varones con SXF, ya a los 9 meses, presentan un retraso en los hitos del desarrollo motor y alteraciones en el tono muscular y en la coordinación motora. A los 24 meses muestran un persistente retraso en el desarrollo del habla y alteraciones comportamentales, aunque no suelen ser diagnosticados hasta un año más tarde. Cuando entran en preescolar muestran una gran variabilidad en su desarrollo: el desfase suele oscilar de un tercio a la mitad por debajo de lo esperado en un desarrollo normotípico. El lenguaje expresivo está más afectado que el receptivo, las puntuaciones en el funcionamiento motor y adaptativo son relativamente más altas comparadas con las 
funciones comunicativas y cognitivas. El desarrollo adaptativo tiene un crecimiento significativo hasta los 10 años, aunque es más lento a partir de los 5 años. Después de los 10 años se producen descensos en las puntuaciones de CI y pérdidas de lo conseguido durante los años anteriores. Desde los años de preescolar los niños presentan déficits en el lenguaje conversacional con un incremento progresivo de las discrepancias entre el nivel de lenguaje y la edad cronológica. El SXF es un factor de riesgo de tener autismo, ya que su sistema nervioso está poco modulado (hiperexcitación y problemas con la inhibición y la habituación) (Reiss y Dant, 2003).

En las mujeres existe una gran variabilidad en su desarrollo. Las que tienen mutación completa muestran alteraciones medias o moderadas y las que tienen premutación muestran un desarrollo parecido al habitual. En preescolar y edad escolar la presencia de ansiedad social, timidez y comportamiento evitativo es un factor de riesgo de contraer depresión en la preadolescencia y adolescencia, y es la principal manifestación del riesgo "genético" de la mutación FMR1 en mujeres. Lo mismo que en los varones, el funcionamiento cognitivo y adaptativo continúa decreciendo a lo largo del desarrollo, y los procesos ejecutivos (memoria de trabajo, inhibición y planificación) no se desarrollan en la adolescencia según lo esperado (Reiss y Dant, 2003).

\section{Perfil cognitivo}

La mayoría de los hombres con SXF y las mujeres sintomáticas presentan una disfunción en la integración sensorial, discapacidad intelectual con puntuaciones de CI que disminuyen con la edad, desórdenes en los procesos cognitivos y viso-espaciales, y trastornos del lenguaje y de la comunicación (Artigas-Pallarés, 2002; Reiss y Dant, 2003; Castro-Volio y Cuenca-Berger, 2005).

\section{Integración sensorial}

Los afectados por el SXF presentan una disfunción de la integración sensorial. Tienen problemas para recibir la información de cada uno de los sentidos, interpretarla e integrarla. No pueden modular, discriminar, coordinar u organizar sensaciones de forma adaptativa. Por todo ello, tienen problemas para procesar la información visual, táctil, auditiva y olfativa (Artigas-Pallarés et al., 2001; Goldson, 2001).

Presentan una respuesta exagerada a los estímulos sensoriales y una defensa sensorial que les conduce a evitar las sensaciones (Artigas-Pallarés et al., 2001; Goldson, 2001). Se sienten inseguros al utilizar información sobre distancias y relaciones espaciales, y pueden perder el equilibrio; evitan la mirada de los interlocutores, ya que el contacto visual es demasiado intenso; se sienten agobiados cuando reciben un abrazo o caricia, son balanceados o tomados en brazos; y les incomoda una determinada textura de ropa, o la presión que la prenda ejerce sobre su cuerpo. Igualmente, los estímulos sonoros pueden representar una sensación desagradable de la que tienen que huir. La experiencia de acudir a un sitio con aglomeraciones puede resultar insopor- 
table al recibir un exceso de estímulos: ruido, movimiento, contacto e imágenes. Los olores y los sabores también pueden provocar malestar o evitación. Una sensación molesta para otras personas puede resultarles insoportable (Artigas-Pallarés et al., 2001). La hipersensibilidad a los estímulos puede estar relacionada con una excesiva interconexión o un exceso de ramas dendríticas (Artigas-Pallarés et al., 2001).

La dificultad en el procesamiento de la información sensorial de los niños con SXF les impide dominar su entorno y les provoca numerosas dificultades en las actividades de la vida diaria, en el aprendizaje, en su participación en la escuela, en la realización de actividades motoras y en las interacciones sociales con los niños de su misma edad y con los adultos (Goldson, 2001).

\section{Intervención educativa}

En el ámbito educativo sería conveniente ofrecerles una estimulación sensorial controlada. Es aconsejable evitar una iluminación intensa y controlar la cantidad de estímulos visuales (fotografías, carteles, colorido de las paredes...); reducir los ruidos innecesarios y proporcionarles una zona para calmarse; evitar los olores químicos fuertes (productos de limpieza, perfumes...) y potenciar los olores naturales de flores; no forzar el contacto físico con el niño y utilizar masajes suaves para ayudar a descargar las tensiones acumuladas; fomentar la visita a la cocina para familiarizarse con los alimentos que vayan a ingerir; e informarles de los movimientos que se requieren para realizar una actividad determinada.

Aunque no existen ensayos clínicos que nos aporten evidencias científicas, algunos autores proponen la utilización de la terapia de integración sensorial desarrollada por la doctora Jean Ayres, terapeuta ocupacional y doctora en neurociencias. Esta terapia tiene como objetivo mejorar la eficacia del SNC en la interpretación y uso de la información sensorial y potenciar su funcionalidad. La terapia es individualizada y se ajusta a los puntos fuertes y débiles de cada niño; asimismo, se utilizan las tendencias espontáneas del niño para desarrollar las capacidades normales de integración sensorial a través de respuestas adaptativas al entorno (Goldson, 2001; Ayres, 2008).

\section{Cognición}

La discapacidad intelectual la presentan la totalidad de los varones con mutación completa y únicamente el $70 \%$ de las mujeres con mutación completa (FerrandoLucas et al., 2003).

\section{Hombres}

El nivel de funcionamiento cognitivo exhibido por los varones con SXF varía desde un funcionamiento normal o bordeline con dificultades de aprendizaje, hasta discapacidad intelectual severa. Este nivel está relacionado con la cantidad de FMRP 
producido por cada individuo. Cuando hay una mutación completa, la producción de FMRP es muy baja o está ausente, lo que causa déficits cognitivos generalizados y un funcionamiento ejecutivo deficiente. Los hombres están más afectados que las mujeres, pero ambos muestran descensos de CI a medida que envejecen. Los descensos en las habilidades cognitivas se producen en todas las áreas: razonamiento verbal, habilidades visuales/abstractas, habilidades cuantitativas, memoria a corto plazo -tanto en la memoria auditiva como en la visual-y atención. Al mismo tiempo se producen descensos en todas las habilidades adaptativas: comunicación, vida diaria y socialización (Visootsak et al., 2005).

Tienen afectada la memoria auditiva a corto plazo, el procesamiento secuencial de la información y la atención. En contraste, algunas destrezas cognitivas son relativamente fuertes, como el procesamiento simultáneo de la información y la memoria a largo plazo para aprender información. El procesamiento simultáneo conlleva la integración y la síntesis de los estímulos como un todo unificado en la resolución de problemas, mientras que el secuencial lo hace paso a paso (Abbeduto et al., 2007). Las fortalezas en el razonamiento verbal se circunscriben al etiquetado sencillo, vocabulario y comprensión verbal (Visootsak et al., 2005).

Al margen de la capacidad intelectual, los varones presentan déficits en las funciones ejecutivas, que se manifiestan en dificultades en la atención, en la planificación y consecución de objetivos, en la memoria de trabajo y en la capacidad de inhibir respuestas inapropiadas (Artigas-Pallarés et al., 2001); también en la habilidad espacial y en la memoria visual (Visootsak et al., 2005).

En la memoria de trabajo muestran déficits en el bucle fonológico y en la agenda viso-espacial, aunque las deficiencias fonológicas son significativamente mayores que las viso-espaciales. Estas deficiencias se mantienen en todo tipo de tareas de baja, media o alta complejidad (Baker et al., 2011). El estudio de la memoria está todavía en sus primeras etapas, pero sus resultados pueden ser útiles para diseñar programas de rehabilitación más adecuados que tengan en cuenta cómo estos déficits pueden afectar a los aprendizajes escolares, a la pobre conciencia fonológica, a los déficits en el procesamiento secuencial y a las funciones de la memoria a largo plazo atenuadas (Johnson-Glenberg, 2008).

Los varones con SXF presentan modelos de activación cerebral diferentes a las personas con un funcionamiento normotípico en la memoria de trabajo y en los procesos viso-espaciales (Menon et al., 2000; Kwon et al., 2001). En las personas sin alteraciones se activa la corteza prefrontal con regiones adicionales cuando se dan las dos condiciones (memoria de trabajo y procesos viso-espaciales); sin embargo, en el SXF sólo se activan zonas dispersas de la corteza prefrontal y parietal en una única tarea y no se incrementa esta actividad cuando se dan las dos condiciones (Menon et al., 2000; Kwon et al., 2001).

Intervención educativa

Como las personas afectadas con el síndrome del X Frágil procesan la información visual mejor que la auditiva, debemos utilizar fundamentalmente la vía visual 
para el acceso a la información. Del mismo modo, al tener el procesamiento simultáneo menos afectado que el secuencial, tendrán problemas en la fonética y decodificación de las palabras, en la identificación de los componentes de un problema, en la interpretación de las partes de un diseño o dibujo, en la comprensión de las reglas y seguimiento de órdenes verbales, en el recuerdo de detalles y en la información presentada de forma temporal paso a paso. Son más competentes, si no existe una discapacidad intelectual asociada, en el reconocimiento visual de las palabras, en la comprensión de los principios globales de las matemáticas y en la interpretación de mapas, diagramas o cartas. Se deben priorizar aquellas tareas que impliquen un procesamiento simultáneo y evitar aquellas que impliquen un procesamiento secuencial (Hodapp y Dykens, 2004). Por este motivo, el método de lectura más idóneo será el global.

\section{Mujeres}

Las mujeres con mutación completa están menos afectadas que los hombres. Aproximadamente un $70 \%$ de las mujeres con mutación completa tienen una puntuación de CI menor a 85, y presentan una discapacidad intelectual bordeline o moderada mientras que las mujeres con premutación tienen un CI normal. Las fortalezas y debilidades de las mujeres son similares a las de los hombres.

Block et al. (2000) hallaron diferencias significativas en el procesamiento cognitivo y en el procesamiento visual entre las mujeres con mutación completa y las mujeres con premutación. Las mujeres con mutación completa tuvieron puntuaciones más bajas en velocidad de procesamiento viso-motor, discriminación y memoria visual, constancia de la forma, percepción figura/fondo, cierre visual y capacidad de análisis y de síntesis.

Las mujeres con SXF presentan déficits en la inhibición e inicio de movimientos sacádicos voluntarios, en la generación de movimientos sacádicos anticipatorios apropiadamente programados y en la desactivación de la fijación, lo que indica una alteración en el área visual frontal, posiblemente en los ganglios basales y en menor grado en el córtex prefrontal dorso lateral. Estas alteraciones de los movimientos oculares pueden ayudarnos a entender las alteraciones en las funciones ejecutivas y visoespaciales de las mujeres con SXF (Lasker et al., 2007).

Las mujeres con mutación completa tienen más dificultades que las que tienen premutación en aritmética y son capaces de realizar tareas de completar dibujos (Visootsak et al., 2005). En las tareas matemáticas presentan una activación cerebral anormal. En las personas sin alteraciones, cuando se incrementa la dificultad de las tareas de matemáticas se produce una activación del lóbulo parietal, córtex prefrontal y cerebelo; sin embargo, en las mujeres con SFX sólo se activa la zona prefrontal (Menon et al., 2000; Rivera et al., 2002).

Aunque no muestren discapacidad intelectual, las mujeres con SXF presentan dificultades en las matemáticas, por su falta de competencia en el conocimiento conceptual de las matemáticas y en el conocimiento procedimental que se utiliza en la resolución 
de problemas, que se apoya en múltiples sistemas cognitivos, incluyendo controles ejecutivos (memoria de trabajo, atención, inhibición...), lenguaje y procesos viso-espaciales (Murphy et al., 2006).

Las niñas con SXF manifiestan fortalezas relativas en contar de memoria y en la representación escrita de números (pueden leer y escribir números de uno o dos dígitos); sin embargo, tienen más problemas en aplicar reglas de cálculo, en formar conjuntos de menos de 10, en la correspondencia uno a uno, en la constancia del número, en contar puntos dispersos y en comparar magnitudes. Las fuertes habilidades memorísticas de las niñas con SXF sugieren que la recuperación de las operaciones matemáticas por sí sola no sería problemática, pero sí la comprensión y aplicación correcta de las operaciones matemáticas (Mazzocco, 2001; Murphy et al., 2006).

Las mujeres también presentan dificultades en algunas tareas con un componente visual espacial, tales como identificación de rectángulos dentro de una matriz de diferentes formas y juzgar longitudes relativas a los objetos representados; sin embargo, en tareas más complejas tienen menos problemas. Es posible que este bajo rendimiento en los ítems más simples e iniciales refleje una dificultad de orientación para una nueva tarea, lo que implicaría una disfunción en los procesos ejecutivos más que dificultades viso-espaciales (Murphy et al., 2006).

\section{Intervención educativa}

Las mujeres tienen afectada la cognición visual (percepción, atención y memoria) y los procesos ejecutivos, lo que produce dificultades de aprendizaje (lectura y matemáticas). Las alteraciones en los movimientos sacádicos de los ojos afectan a la velocidad y comprensión lectora. Además de aplicar estrategias para mejorar los procesos lectores, y la resolución de problemas, se deben incluir técnicas de entrenamiento visual para mejorar los movimientos sacádicos.

\section{Lenguaje}

Los varones tienen más afectado el lenguaje que las mujeres: es el reflejo de las diferencias en el funcionamiento intelectual de ambos sexos. Existen pocos trabajos sobre el lenguaje en mujeres con SXF. Asimismo, los afectados que presentan comorbidad de SXF con autismo muestran grave retraso en el desarrollo del lenguaje. El perfil lingüístico del SXF es diferente del perfil del SD (Visootsak et al., 2005; Lewis et al., 2006; Abbeduto et al., 2007).

Los varones con premutación o mutaciones intermedias muestran dificultades en el componente pragmático del lenguaje y tienen riesgo de tener retrasos en el desarrollo del vocabulario receptivo y expresivo; y dificultades en las habilidades motoras, en la fluencia y en la entonación (Visootsak et al., 2005).

Los varones con SXF con mutación completa muestran retrasos en el desarrollo del lenguaje: la adquisición del lenguaje expresivo es más lenta que la del receptivo. La 
discrepancia entre ellos se incrementa a medida que crece (Visootsak et al., 2005). Presentan retrasos con relación a los sujetos de su misma edad en los hitos del desarrollo del periodo prelingüístico y en sus progresos en todos los dominios del habla y del lenguaje, vocabulario, morfosintaxis y pragmática (Visootsak et al., 2005).

\section{Desarrollo fonético y fonológico}

Aunque los varones con SXF presentan retrasos en el desarrollo del habla, tienen puntuaciones similares a los sujetos con la misma edad mental: a) en el dominio correcto de las consonantes de desarrollo temprano, medio y tardío; b) en los procesos fonológicos de sustitución (frontalización velar y palatal, sonorización/ensordecimiento y simplificación fricativa y líquida), de estructura silábica (supresión de consonantes finales, reducción de grupos silábicos y cambios en la estructura silábica -reducción, omisión y reduplicación-) y de asimilación (asimilación nasal y velar); y c) en la proporción de proximidad de las palabras completas. Asimismo, su habla es menos inteligible por problemas de prosodia y de fluidez (Roberts et al., 2005; Barnes et al., 2009).

Sin embargo, obtienen peores resultados que los sujetos con el mismo nivel de desarrollo mental: a) en la estructura de los labios, mandíbula, maxilar, dientes, velofaringe y laringe y especialmente, en la lengua (estructura oral); y b) en el movimiento de los labios, lengua y velofaringe (funcionamiento oral). Tienen más problemas que los varones con SD en los movimientos de la lengua. Asimismo, obtienen similares resultados en las funciones del habla (repetición de fonemas y palabras y coordinación de movimientos para su articulación) (Barnes et al., 2006). Los varones con SXF obtienen mejores resultados en funciones del habla que en las orales, indicando un mayor retraso en las funciones orales no acompañadas por la palabra. Por el contrario, los sujetos con SD puntúan más alto en las funciones orales que en las del habla. Los varones con SXF y SD repiten las palabras de una única sílaba con mayor precisión que las que tienen múltiples sílabas, mientras que los varones con un desarrollo estándar producen ambos tipos de palabras con la misma precisión (Barnes et al., 2006).

En los individuos con mayor funcionalidad, el habla puede ser rápida, no fluida y caracterizada por "saturación": tasa alta de palabras con repeticiones de sonidos, palabras y frases, ecolalia y, en ocasiones, un habla ilegible, con arrastre de palabras y desorganizada (Visootsak et al., 2005).

\section{Desarrollo semántico y morfosintáctico}

En las personas con SXF, el vocabulario y la morfosintaxis receptiva correlacionan con la cognición no verbal y presentan tasas similares de desarrollo. El curso de la morfosintaxis expresiva es menos claro: aparece un perfil asincrónico, coexistiendo algunos logros morfosintácticos con otros más atrasados. Producen enunciados generales más cortos, así como frases sustantivas y predicativas y estructuras sintácticas 
menos complejas en contextos conversacionales y narrativos, aunque son mejores que las de los individuos con SD (Abbeduto et al., 2007; Roberts et al., 2007; Price et al., 2008; Finestack y Abbeduto, 2010; Finestack et al., 2012; Finestack et al., 2013).

\section{Desarrollo pragmático}

Las personas con SXF presentan perseverancia verbal y problemas en la comunicación referencial, siendo éstos aspectos especialmente problemáticos (Abbeduto et al., 2007; Price et al., 2008; Finestack et al., 2012; Finestack et al., 2013). Tienen un lenguaje atípico en las interacciones verbales, con expresiones perseverativas (introduciendo sus tópicos favoritos una y otra vez), con cuestiones, respuestas y comentarios que no son pertinentes con el tema de conversación, y que es más acentuado cuando hay comorbilidad con autismo y respecto a sujetos con SD. El lenguaje perseverante puede ser debido a: las anomalías en los lóbulos frontales, que provocan un déficit en la inhibición; al déficit de atención e hiperactividad; y a la alteración en la regulación del sistema nervioso autónomo y aumento de los niveles de cortisol, que son los causantes de la hiperexcitación y ansiedad social (Visootsak et al., 2005; Abbeduto et al., 2007; Roberts et al., 2007; Finestack et al., 2013).

\section{Limitaciones en las investigaciones}

En cuanto a las limitaciones en la investigación sobre el desarrollo del lenguaje podemos señalar (Abbeduto et al., 2007):

- Existen pocas investigaciones sobre el desarrollo prelingüístico de los niños y niñas con SXF y estas investigaciones son imprescindibles para desarrollar intervenciones efectivas; tampoco existen investigaciones sobre la utilización de los sistemas de comunicación no verbales en personas con SXF.

- Las investigaciones sobre el desarrollo del vocabulario se han centrado fundamentalmente en evaluar el dominio de un vocabulario concreto, dejando por explorar dominios específicos del léxico y el proceso mediante el cual las personas con SXF aprenden nuevas palabras. Los estudios sobre el desarrollo de la morfosintaxis se han limitado a medidas más bien amplias, como la longitud de las frases, lo cual puede enmascarar el perfil de dificultades del SXF.

- No se ha prestado atención al modo en que se han elegido las muestras de lenguaje y, sin una adecuada estandarización de las muestras, las investigaciones no son fiables. Tampoco se ha estudiado la secuencia en la adquisición de la morfosintaxis y los errores previos que han cometido, que son importantes para entender el proceso de aprendizaje.

- En la investigación sobre el lenguaje pragmático es necesario ir más allá de un enfoque que se centre en las dimensiones lingüísticas de la comunicación para examinar las características gestuales y las prosódicas del proceso de comunicación. 


\section{Intervención educativa}

Es importante favorecer situaciones en que el alumnado sienta la necesidad de comunicarse, reforzar todo intento comunicativo, crear oportunidades de interacción comunicativa, fomentar el uso adecuado del lenguaje de forma natural y regular su velocidad. Del mismo modo, hay que utilizar recursos visuales para la adquisición del vocabulario y organización de las frases y realizar ejercicios (discriminación auditiva, respiración, soplo, fonación, labios, lengua y mandíbula) para mejorar la pronunciación correcta de los fonemas (Del Barrio, Castro y Buesa, 2006).

En los casos en que hay comorbilidad con autismo se pueden utilizar la Comunicación Total de Benson Schaeffer o el Sistema de Comunicación por Intercambio de Imágenes PECS.

\section{Dificultades en lectura y escritura}

Entre las causas de las dificultades en la lectura y escritura de los varones con SXF se destacan: las alteraciones del lenguaje, que oscilan desde el tartamudeo leve a problemas graves en las habilidades lingüísticas básicas; las dificultades práxicas, que interfieren en la correcta articulación de los fonemas y en la motricidad fina y coordinación viso-motora; las dificultades en el procesamiento secuencial y las fortalezas en el procesamiento simultáneo, lo que repercute negativamente en el procesamiento de la información verbal y los problemas en la percepción auditiva (Fernández-Lozano et al., 2011). Entre los problemas severos cabe destacar la incapacidad de pronunciar palabras de forma clara, la dificultad de escribir y usar la gramática de manera correcta; y la torpeza para comunicarse de forma significativa.

Estas dificultades son más usuales en los hombres que en las mujeres; éstas suelen manejar un vocabulario adecuado, aprenden palabras nuevas de forma rápida, reconocen los sonidos de manera precisa y utilizan la gramática de acuerdo con su edad; sin embargo, la ansiedad social y la timidez les impiden comunicarse de forma apropiada (Roberts et al., 2005; Fernández-Lozano et al., 2011).

La lectura es uno de los aspectos relativamente fuertes en las mujeres, ya que suelen descodificar muy bien y poseen buenas habilidades de comprensión lectora, aunque rinden mejor cuando hacen interpretaciones literales que cuando hacen inferencias (Fernández-Lozano et al., 2011). Por el contrario, los varones muestran una gran disparidad: existe un pequeño grupo que son incapaces de leer; un grupo más grande que es capaz de leer palabras aisladas, frases y textos cortos; $y$, por último, un grupo pequeño que alcanza cierto nivel lector y es capaz de leer capítulos cortos de un libro, revistas o periódicos (Fernández-Lozano et al., 2010; Fernández-Lozano et al., 2011).

Los niños con SXF tienen problemas en el desarrollo fonológico, particularmente en la conciencia fonológica (impidiéndoles recordar secuencias verbales mientras leen y comprenden textos cortos) y en la lectura de pseudopalabras. Asimismo, tienen problemas en la percepción auditiva, como discriminar sonidos, reconocer sonidos fuera de las palabras, mezclar sonidos para formar palabras, silabear y reconocer si 
dos palabras riman. Si no son capaces de separar los sonidos que constituyen una palabra o mezclar sonidos para componer una palabra, es difícil que puedan aprender a leer por el método fonético (Fernández-Lozano et al., 2010; Fernández-Lozano et al., 2011).

Las personas con SXF también tienen problemas en la escritura (particularmente los varones) ya que pueden tener dificultades en comprender la tarea, en el lenguaje receptivo, en las habilidades motoras finas y en la memoria visual suficiente para deletrear, secuenciar letras, palabras e ideas. Estas dificultades se agravan cuando hay déficits cognitivos moderados y severos; asimismo, cuando tienen dificultades para copiar (disgrafía) que implican dificultades en la planificación y ejecución motora, para la formulación de oraciones y para la sintaxis (Fernández-Lozano et al., 2010; Fernández-Lozano et al., 2011).

\section{Intervención educativa}

Se propone para el aprendizaje de la lectura (Fernández-Lozano et al., 2010; Fernández-Lozano et al., 2011) el uso de:

a) Un método global, basado en la memoria visual de las palabras.

b) El entrenamiento fonológico, a través de: estímulos fonéticos iniciales (se proporciona el primer sonido de la palabra); palabras-estímulo que riman (se presenta una palabra que rima con otra con el objeto de que el niño recuerde la palabra); palabras-estímulo asociadas (se propone una palabra que está conectada de alguna manera con otra por su significado); palabras-estímulo en secuencias (se propone una palabra que puede aparecer en una secuencia); estímulos visuales escritos, pictóricos, icónicos y en color; y estímulos gestuales (usar un gesto para evocar una palabra).

c) El fomento de la comprensión lectora a través de la lectura funcional (leer listas del mercado, leer nombres de productos, repetir los pasos para la elaboración de un plato, interpretar horarios e itinerarios del transporte público, leer los programas de la TV que les gustan y los títulos de las películas...).

d) También hay niños con SXF que son hiperléxicos, con mejor habilidad para descodificar que para leer. La comprensión literal de lo leído puede ser una meta en estos casos.

Aunque la adquisición de la escritura es difícil en los niños con SXF, no es imposible. Se pueden utilizar estrategias como: a) escribir palabras que tengan importancia para ellos (su nombre, el de sus hermanos y padres...); b) desarrollar las habilidades viso-motoras, trazar letras en la arena, enseñar a coger el lápiz, utilizar materiales como la plastilina...; c) enseñarles a escribir oraciones sencillas en las que puedan rellenar espacios en blanco o darles el comienzo de una frase para que la continúen, e iniciarles en la escritura de párrafos, empezando con una oración y luego añadir tres 
que completen el tema de la primera oración; y d) mejorar el deletreo con listas de palabras adecuadas a su nivel cognitivo; con actividades de rellenado de espacios en blanco que mejoran la memoria visual, y con actividades para remarcar el número de sílabas de las palabras, la secuencia de las sílabas y el número de letras (FernándezLozano et al., 2010; Fernández-Lozano et al., 2011).

\section{Psicopatología y conductas inadaptativas}

Las personas con SXF se caracterizan por: falta de atención e hiperactividad; ansiedad y miedo a los animales, situaciones o lugares; timidez y comportamiento vergonzoso; apego a los adultos; estereotipia de aleteo de manos; evitación de la mirada; repetición de ciertos actos una y otra vez; preocupación por el orden y la limpieza; tozudez; y agresividad (Artigas-Pallarés y Brun-Gasca, 2004).

Algunos problemas conductuales están ligados a las deficiencias cognitivas, fundamentalmente en dos desórdenes que suelen estar ligados al SXF: el déficit de atención e hiperactividad, y trastorno del espectro del autismo (Cornish et al., 2008).

En el caso de las mujeres con premutación, los rasgos clínicos más destacados son: timidez, ansiedad y fobia social, depresión e inestabilidad emocional en aproximadamente un 25\% de las mujeres portadoras (Artigas-Pallarés et al., 2001; Cornish et al., 2008). Aunque ha habido un debate respecto a que los problemas emocionales de las mujeres con premutación pueden ser debidos al estrés de la crianza de un hijo con SXF, existen algunos estudios que lo relacionan con el nivel de repeticiones CGG (más de 100 o menos de 100) y por tanto de los niveles de FMRP (Hessl et al., 2005; Cornish et al., 2008).

En hombres con premutación, se observa una paradójica combinación de desinhibición social y amabilidad, con dificultades para mostrar emociones y sentimientos, y con problemas de percepción social y empatía: no saben descifrar los sentimientos de los otros y tienen dificultad para hacer amigos (Cornish et al., 2005). Parece que hay una activación de la amígdala en estos sujetos (Hessl et al., 2005).

\section{Déficit de atención e hiperactividad}

Las personas con SXF presentan déficit de atención e hiperactividad en la mayoría de los niños y en el 30\% de las niñas. Los síntomas suelen aparecer a partir de los 2 años de edad, tienden a mejorar después de la pubertad y suelen ser los rasgos más importantes en niños con un funcionamiento elevado (inteligencia normal). En los casos de premutación pueden presentar síntomas de hiperactividad (Calvani et al., 2001; Sullivan et al., 2006; Sullivan et al., 2007; Beltrán et al., 2011).

Las personas con SXF suelen presentar dificultades de atención, distracción, agitación, inquietud e impulsividad. Son más frecuentes los síntomas de falta de atención (100\% de los casos) que los de impulsividad (70\%) (Artigas-Pallarés y Brun-Gasca, 2004; Beltrán et al., 2011) y predomina el subtipo inatento (Cornish et al., 2008). Suelen 
tener una relativa fortaleza en la atención sostenida, una moderada dificultad en atención selectiva y una profunda dificultad en las destrezas que requieren control inhibitorio. Esta última dificultad afecta al desarrollo de destrezas que requieren el control de la atención (lenguaje, memoria de trabajo...), en contraste con otras destrezas que no requieren el control atencional (vocabulario, memoria a largo plazo y reconocimiento facial) en las que son relativamente fuertes (Cornish et al., 2008). Los déficits en el control inhibitorio y sus efectos a lo largo del ciclo vital son muy frecuentes en los varones con premutación y son los precursores del desarrollo de déficits cognitivos severos, como demencia o FXTAS (Síndrome de temblor-ataxia) (Cornish et al., 2008).

Estas características pueden ser atribuibles a alteraciones en el cerebelo y en los circuitos frontoestriales. Las personas afectadas con SXF no son capaces de modular la activación del córtex prefrontal y parietal en respuesta a una demanda de la memoria de trabajo (Beltrán et al., 2011).

La medicación estimulante de los sistemas de los neurotransmisores de dopamina y norepinefrina (metilfenidato, dextroanfetaminas, pemolina) mejora la atención y la coordinación viso-motora y reduce la hiperactividad. Esta medicación ayuda a un porcentaje entre un $60 \%$ y un $70 \%$ de pacientes con SXF en edad escolar. Es menos efectiva en adolescentes o preescolares, y tiene efectos secundarios, como incremento de presión arterial y del ritmo cardíaco, pérdida del apetito, problemas de sueño y empeoramiento de los tics motores preexistentes. Estos síntomas se agudizan cuando se aumentan las dosis, así como la irritabilidad y las rabietas cuando cesa la medicación. Otra substancia que se está utilizando para mejorar la hiperactividad del SXF es la L-acetilcarnitina, bajo la hipótesis de que ésta puede preservar mejor la actividad cerebral de las personas con SXF (Calvani et al., 2001).

\section{Intervención educativa}

Para mejorar la atención se debe: a) proporcionar un entorno que elimine las distracciones (situar a la persona de espaldas a la puerta, colocar cortinas en ventanas, seleccionar el material necesario, trabajar en grupos reducidos y utilizar material visual); b) dar información sobre qué estímulos debe atender y cuáles ignorar y diseñar tareas atractivas y de su interés; c) aumentar progresivamente los periodos atencionales y proporcionar tiempos de descanso; y d) introducir paulatinamente estímulos nuevos y agradables, e intercalarlos con otros conocidos (Medina, 2014; Del Barrio, Castro y Buesa, 2006).

\section{Trastorno del espectro del autismo}

Entre un $2 \%$ y un $6 \%$ de los niños con trastorno del espectro del autismo (en adelante TEA) presentan la mutación genética del SXF. De hecho, la mayoría de los niños con SXF muestran conductas que también se dan en niños con TEA, aunque sólo de un $20 \%$ a $30 \%$ cumplen los criterios diagnósticos del trastorno autista ya que existen 
elementos diferenciales. Este porcentaje se incrementa con la edad en varones con mutación completa (García-Nonell et al., 2006; Cornish et al., 2008; Beltrán et al., 2011).

Son rasgos comunes frecuentemente: el aleteo; el morderse la mano, la defensa táctil; el lenguaje repetitivo, estereotipado y con dificultades pragmáticas; el rechazar los cambios en la rutina. Sin embargo, presentar estos rasgos no quiere decir que tengan un trastorno del espectro del autismo, ya que en muchos casos no muestran los déficits sociales típicos del autismo. Las razones de estos comportamientos son diferentes a las de las personas con TEA (García-Nonell et al., 2006).

Por ejemplo, la ausencia de contacto ocular en el autismo está producida por la incapacidad para comprender las situaciones sociales y por la falta de deseo para comunicarse, mientras que en el SXF es por la gran sensibilidad hacia los estímulos sensoriales y por la ansiedad social. Suelen evitar la mirada, rechazar el contacto físico y tener rabietas cuando hay un exceso de estimulación. Su mirada es comunicativa, aunque eviten el contacto ocular; esta evitación es selectiva, siendo menos frecuente ante personas conocidas que ante las desconocidas. También son extremadamente tímidos y manifiestan ansiedad ante situaciones novedosas, mostrando estereotipias como el aleteo y morderse la mano. Aunque se muestran tímidos y angustiados ante situaciones de interacción social, éstas no les son indiferentes, ni tampoco las rechazan, sino que incluso las pueden provocar (García-Nonell et al., 2006; Cornish et al., 2008; Beltrán et al., 2011).

Posiblemente la ansiedad es uno de los síntomas nucleares del SXF, y se debe a alteraciones neuroanatómicas y disfunciones neuroendocrinas hipotálamica-hipofisaria-adrenal. De hecho, tanto los niños como las niñas con SXF tienen una concentración de cortisol superior a la normal. En ambos casos suelen tener el mismo nivel de cortisol basal, aunque, ante una situación estresante, los niños segregan más que las niñas (Artigas-Pallarés y Brun-Gasca, 2004).

La conducta de las personas con SXF suele ser obsesiva; a pesar de ello, muestran una buena capacidad de imitación, de comprensión del lenguaje y de memoria visual (García-Nonell et al., 2006; Beltrán et al., 2011).

La incapacidad para entender las creencias e intenciones de los otros tiene también razones diferentes en el autismo que en el SXF. En el autismo, existe una patología grave del desarrollo de la intersubjetividad, ligada a un déficit sustancial en la teoría de la mente. La teoría de la mente hace referencia a la capacidad de atribuir pensamientos, intenciones y emociones a otras personas, basándose en el contexto e interfiriendo a partir de la propia mente y de las propias experiencias de vida (Rondal, 2007). En el caso del SXF, esta incapacidad se asocia más a las dificultades en el procesamiento de la información que afectan a la memoria de trabajo (sobre todo, a aquellas que requieren atención y capacidad ejecutiva) que al núcleo deficitario de la teoría de la mente, que sería la causa en el autismo (Cornish et al., 2008).

\section{Intervención educativa}

Respecto a los problemas en los procesos ejecutivos, especialmente si existe comorbilidad con autismo, es imprescindible proporcionarles una enseñanza que facilite: 
la estructura física del aula (organización por rincones delimitados visualmente), la secuencia de eventos (mediante las agendas), la organización de tareas individuales mediante claves visuales y la conexión del conjunto de las mismas dentro de una secuencia de actividades, lo que permite al alumnado saber cómo comportarse y anticipar lo que va a ocurrir (Ferrer, Arocas y García, 2012). Además de la anticipación de los sucesos, se debe evitar el contacto ocular y físico; el alumno debe mantenerse distante del grupo y de lugares multitudinarios, y llevar algún objeto personal, todo lo cual puede ser útil para manejar la ansiedad social (Medina, 2014).

También, son útiles las técnicas de modificación de conducta como el refuerzo positivo, el encadenamiento, el tiempo muerto, la economía de fichas y el modelado. Debemos mostrar siempre los comportamientos que se esperan de ellos (guiones de conducta) y la conducta alternativa, ser claros y contundentes con las órdenes y frases, ser constantes en las consecuencias ante la aparición de la conducta y disponer de normas de clase en un lugar visible para mostrarlas cuando sea necesario. Las historias sociales acompañadas de dibujos e ilustraciones pueden ser útiles para que el alumnado comprenda las relaciones sociales y los códigos convencionales de conducta en situaciones de relaciones interpersonales (Martos-Pérez y Llorente-Comí, 2013).

\section{Conclusiones}

Existe una gran variabilidad en el fenotipo conductual de las personas con SXF y, por lo tanto, en las estrategias educativas aplicables.

La severidad de las dificultades de aprendizaje y comportamentales del alumnado con SXF está relacionada con el estado de mutación y con el sexo. Asimismo, cuanto mayores son las dificultades de aprendizaje, aumentan y más graves son las manifestaciones psicopatológicas.

En la actualidad, los tratamientos para el Síndrome X frágil no actúan sobre las causas, sino para mejorar los síntomas y deben abordarse por un equipo coordinado de diferentes especialistas.

Los profesionales deben tener en cuenta las características cognitivas y conductuales para poder guiar el aprendizaje de las personas con SXF para organizar la información y actuar sobre la misma de una forma efectiva teniendo en cuenta los siguientes aspectos: atención, hiperactividad e impulsividad; aprendizaje; habla y lenguaje; incapacidad para procesar la información sensorial de manera efectiva; habilidades motoras escasamente desarrolladas, y problemas de comportamiento. Así, deben tener en cuenta: los problemas de integración sensorial; la pobre memoria auditiva y la conciencia fonológica; los déficits en la atención selectiva, en el procesamiento secuencial de la información y en las funciones ejecutivas (control atencional, planificación, consecución de objetivos, memoria de trabajo y capacidad de inhibir respuestas inapropiadas); y la memoria a largo plazo atenuada.

Las estrategias educativas tienen que basarse en las fortalezas: procesamiento simultáneo mejor que el secuencial; procesamiento de la información visual mejor que la auditiva; atención sostenida mejor que la selectiva; la memoria a largo plazo para 
aprender información; y el vocabulario receptivo y expresivo. Deben actuar sobre los problemas de aprendizaje en las matemáticas y en el lenguaje oral y escrito, desarrollando metodologías que se centren en las fortalezas para compensar los déficits y controlar los comportamientos inadaptativos.

En esta labor es imprescindible la colaboración de los logopedas escolares para mejorar el retraso en el desarrollo del lenguaje en todos los dominios del habla y del lenguaje (vocabulario, morfosintaxis y pragmática). También la de los psicólogos escolares y clínicos, con objeto de ayudar a los padres a entender los retrasos en el desarrollo de sus hijos (que en ocasiones es la tarea más difícil) y controlar los comportamientos psicopatológicos. E igualmente la de los terapeutas ocupacionales, fundamentalmente en dos ámbitos: en el de la escuela, para estructurar el entorno, fomentar la participación, la realización de actividades motoras precisas o no precisas y la interacción con niños de su misma edad y con adultos; y en ciclos educativos superiores, para desarrollar una actividad productiva satisfactoria en función de sus habilidades motoras.

Es imprescindible en el ámbito educativo desarrollar investigaciones aplicadas que permitan determinar las metodologías didácticas más efectivas para las personas con SXF y dirigidas hacia: a) la fijación de la meta, autocontrol, autorreforzamiento y ajuste de metas; b) la prevención de la sobreestimulación, a través de técnicas terapéuticas calmantes y de reforzamiento positivo de la conducta; c) la terapia del habla y del lenguaje; e) la estimulación sensorial; f) la mejora de los procesos cognitivos; g) la modificación del formato instructivo y del plan de estudios; h) la introducción de materiales visuales que el niño pueda usar para aprender nuevas habilidades y rutinas; i) la participación en actividades de pequeños grupos; y j) la modificación del material pedagógico para adecuarlo al nivel de desarrollo del niño.

\section{Referencias bibliográficas}

Abbeduto, L., Brady, N. y Kover, S. T. (2007). Language Development and Fragile X Syndrome: Profiles, Syndrome-Specificity, and Within-Syndrome Differences. Mental Retardation and Developmental Disabilities Research Reviews, 13, 36-46. doi:10.1002/mrdd.20142

Artigas-Pallarés, J. (2002). Fenotipos conductuales. Revista de Neurología, 34 (Supl. 1): S38-S48.

Artigas-Pallarés, J. y Brun-Gasca, C. (2004). ¿Se puede atribuir el fenotipo conductual del síndrome $\mathrm{X}$ frágil al retraso mental y al trastorno por déficit de atención/hiperactividad? Revista de Neurología, 38 (1), 7-11.

Artigas-Pallarés, J., Brun, C. y Gabau, E. (2001). Aspectos médicos y neuropsicológicos del síndrome X frágil. Revista de Neurología Clínica, 2 (1), 42-54.

Artigas-Pallarés, J., Gabau-Vila, E. y Guitart-Feliubadaló, M. (2006). Fenotipos conductuales en el retraso mental de origen genético. Revista de Neurología, 42 (Supl. 1), S15-S19.

Ayres, A. J. (2008). La integración sensorial en los niños: desafíos sensoriales ocultos. Madrid, Spain: Tea Ediciones.

Baker, S., Hooper, S., Skinner, M., Hatton, D., Schaaf, J., Ornstein, P. y Bailey, D. (2011). Working memory subsystems and task complexity in young boys with Fragile X syndrome. Journal of Intellectual Disability Research, 55 (1), 19-29. doi:10.1111/j.13652788.2010.01343.x 
Barnes, E., Roberts, J., Long, S. H., Martin, G. E., Berni, M. C., Mandulak, K. C. y Sideris, J. (2009). Phonological Accuracy and Intelligibility in Connected Speech of Boys with Fragile X Syndrome or Down Syndrome. Journal of Speech, Language and Hearing Research, 52, 1048-1061. doi:10.1044/1092-4388(2009/08-0001)

Barnes, E. F., Roberts, J., Mirrett, P., Sideris, J. y Misenheimer, J. (2006). A Comparison of Oral Structure and Oral-Motor Function in Young Males with Fragile X Syndrome and Down Syndrome. Journal of Speech, Language and Hearing Research, 49, 903-917. doi:10.1044/1092-4388(2006/065)

Beltrán, D. A., Guerra, J. A., Crespo, A. E., López-Muñoz, F. y Álamo, C. (2011). Síndrome de cromosoma X frágil. Toko-Ginecología Práctica, 70 (1), 7-20.

Block, S. S., Brusca-Vega, R., Pizzi, W. J., Berry-Kravis, E., Maino, D. M. y Treitman, T. M. (2000). Cognitive and Visual Processing Skills and Their Relathionship to Mutation Size in Full and Permutation Female Fragile X Carriers. Optometry and Vision Science, 77 (11), 592-599.

Calvani, M., D’Iddio, S., De Gaetano, A., Mariotti, P., Mosconi, L., Pomponi, M. G., Tabolacci, E., Torriolil, M. G., Vernacotola, S. y Neri, G. (2001). El tratamiento con $\mathrm{L}$-aceltilcarnitina del comportamiento hiperactivo de pacientes con el síndrome $\mathrm{X}$ frágil. Revista de Neurología, 33 (Supl. 1), S65-S70.

Castro-Volio, I. y Cuenca-Berger, P. (2005). Trastornos del neurodesarrrollo (síndrome X frágil) y neurodegenerativos (síndrome de temblor/ataxia) asociados al "crecimiento" de un gen. Revista de Neurología, 40 (7), 431-437.

Cornish, K., Kogan, C., Turk, J., Manly, T., James, N., Mills, A. y Dalton, A. (2005). The emerging fragile $\mathrm{X}$ premutation phenotype: Evidence from the domain of social cognition. Brain and Cognition, 57 (1): 53-60. doi:10.1016/j.bandc.2004.08.020

Cornish, K., Turk, J. y Hagerman, R. (2008). The fragile X continuum: new advances and perspectives. Journal of Intellectual Disability Research, 55 (6), 469-482. doi:10.1111/ j.1365-2788.2008.01056.x

Del Barrio, J. A., Castro, A. y Buesa, L. (2006). Síndrome del X Frágil. En FEAPS, Síndromes y apoyos. Panorámica desde la ciencia y desde las asociaciones (pp. 161-189). Madrid: FEAPS.

Di Nuovo, S. y Buono, S. (2011). Behavioral phenotypes of genetic syndromes with intellectual disability: Comparison of adaptive profiles. Psychiatry Research, 189 (3), 440-445. doi:10.1016/j.psychres.2011.03.015

Fernández-Lozano, M. P., Puente-Ferreras, A. y Ferrando-Lucas, M. T. (2010). Síndrome X frágil: Desarrollo e intervención del lenguaje escrito. Revista Chilena de NeuroPsiquiatría, 48 (3), 219-231.

Fernández-Lozano, M. P., Puente-Ferreras, A. y Ferrando-Lucas, M. T. (2011). Lectura y escritura en niños con síndrome X frágil: estrategias de intervención. Anales de Psicología, 47 (3), 808-815.

Ferrando-Lucas, M. T., Banús-Gómez, P. y López-Pérez, G. (2003). Aspectos cognitivos y del lenguaje en niños con síndrome X frágil. Revista de Neurología, 36 (Supl. 1), S137-S142.

Ferrer, A., Arocas, E. y García, J. M. (2012). Intervención psicoeducativa en trastornos del espectro autista. En C. Grau y M. D. GIL (Coords.), Intervención Psicoeducativa en Necesidades Especificas de Apoyo Educativo (pp. 211-242). Madrid: Pearson Educación.

Finestack, L. H. у Aвbeduto, L. (2010). Expressive Language Profiles of Verbally Expressive Adolescents and Young Adults with Down Syndrome or Fragile X Syndrome. Journal of Speech, Language, and Hearing Research, 53 (5), 1334-1348. doi:10.1044/10924388(2010/09-0125) 
Finestasck, L. H., Palmer, M. y Abbeduto, L. (2012). Macrostructural Narrative Language of Adolescents and Young Adults with Down Syndrome or Fragile X Syndrome. American Journal of Speech-Language Pathology, 21 (1), 29-46. doi:10.1044/1058-0360(2011/10-0095)

Finestack, L. H., Sterling, A. M. y Abbeduto, L. (2013). Discriminating Down Syndrome and Fragile X Syndrome based on language ability. Journal of Child Language, 40 (1), $244-$ 265. doi:10.1017/S0305000912000207

García-Nonell, C., Rigau-Ratera, E. y Artigas-Pallarés, J. (2006). Autismo en el síndrome X frágil. Revista de Neurología, 42 (Supl. 2), S95-S98.

Goldson, E. (2001). Integración sensorial y síndrome X Frágil. Revista de Neurología, 33 (Supl. 1), S32-S36.

Hessl, D., Tassone, F., Loesch, D. Z., Berry-Kravis, E., Leehey, M. A., Gane, L. W., Barbato, I., Rice, C., Gould, E., Hall, D. A., Grigsby, J., Wegelin, J. A., Harris, S., Lewin, F., Weinberg, D., Hagerman, P. J. y Hagerman, R. J. (2005). Abnormal elevation of FMR1 mRNA is associated with psychological symptoms in individuals with the fragile $\mathrm{X}$ permutation. American Journal of Medical Genetics Part B: Neuropsychiatric Genetics, 139B: 115-121. doi:10.1002/ajmg.b.30241

Hodapp, R. M. y Dykens, E. M. (2004). Genética y fenotipo conductual en la discapacidad intelectual: su aplicación a la cognición y a la conducta problemática (1. ${ }^{a}$ parte). Revista Sindrome de Down, 21, 134-149.

Hodapp, R. M. y Dykens, E. M. (2005). Genética y fenotipo conductual en la discapacidad intelectual: su aplicación a la cognición y a la conducta problemática (2. ${ }^{a}$ parte). Revista Sindrome de Down, 22, 27-36.

Johnson-Glenberg, M. C. (2008). Fragile X syndrome: Neural network models of sequencing and memory. Cognitive Systems Research, 9, 274-292. doi:10.1016/j.cogsys.2008.02.002

Kwon, H., Menon, V., Eliez, S., Warsofsky, I. S., White, C. D., Dyer-Friedman, J., Taylor, A. K., Glover, G. H. y Reiss, A. L. (2001). Functional Neuroanatomy of Visuospatial Working Memory in Fragile X Syndrome: Relation to Behavioral and Molecular Measures. The American Journal of Psychiatry, 158 (7), 1040-1051. doi:10.1176/appi.ajp.158.7.1040

Lasker, A. G., Mazzocco, M. M. M. y Zee, D. S. (2007). Ocular motor indicators of executive dysfunction in fragile $\mathrm{X}$ and Turner syndromes. Brain and Cognition, 63, 203-220. doi:10.1016/j.bandc.2006.08.002

Lewis, P., Abbeduto, L., Murphy, M., Richmond, E., Giles, N., Bruno, N. y Schroeder, S. (2006). Cognitive, language and social-cognitive skills of individuals with fragile X syndrome with and without autism. Journal of Intellectual Disability Research, 50 (7), 532-545. doi:10.1111/j.1365-2788.2006.00803.x.

Martos-Pérez, J. y Lorente-Comí, M. (2013). Tratamiento de los trastornos del espectro autista: unión entre la comprensión y la práctica basada en la evidencia. Revista Neurología, 57 (1), S185-S191.

Mazzocco, M. M. M. (2001). Math Learning Disability and Math LD Subtypes: Evidence from Studies of Turner Syndrome, Fragile X Syndrome, and Neurofibromatosis Type 1. Journal of Learning Disabilities, 34 (6), 520-533. doi:10.1177/002221940103400605

Medina, B. (2014). El síndrome del X Frágil: identificación del fenotipo y propuestas educativas. Revista Española de Discapacidad, 2 (2), 45-62.

Menon, V., Kwon, H., Eliez, S., TAylor, A. K. y Reiss, A. L. (2000). Functional brain activation during cognition is related to FMR1 gene expression. Brain Research, 877 (2), 367-370. doi:10.1016/S0006-8993(00)02617-2

Menon, V., Rivera, S. M., White, C. D., Glover, G. H. y Reiss, A. L. (2000). Dissociating Prefrontal and Parietal Cortex Activation during Arithmetic Processing. Neuroimage, 12, 357-365. doi:10.1006/nimg.2000.0613 
Murphy, M. M., Mazzoco, M. M. M., Gerner, G. y Henry, A. E. (2006). Mathematics learning disability in girls with Turner syndrome or fragile X syndrome. Brain and Cognition, 61, 195-210. doi:10.1016/j.bandc.2005.12.014

O'Brien, G. (2006). Behavioural phenotypes: causes and clinical implications. Advances in Psychiatric Treatment, 12 (5): 338-348. doi:10.1192/apt.12.5.338

Price, J. R., Roberts, J. E., Hennon, E. A., Berni, M. C., Anderson, K. L. y Sideris, J. (2008). Syntactic Complexity during Conversation of Boys with Fragile X Syndrome and Down Syndrome. Journal of Speech, Language and Hearing Research, 51, 3-15. doi:10.1044/1092$4388(2008 / 001)$

Reiss, A. L. y Dant, C. C. (2003). The behavioral neurogenetics of fragile X syndrome: Analyzing gene-brain-behavior relationships in child developmental psychopathologies. Developmental and Psychopathology, 15: 927-968. doi:10.1017.S0954579403000464

Rivera, S. M., Menon, V., White, C. D., Glasser, B. y Reiss, A. L. (2002). Functional brain activation during arithmetic processing in females with fragile $\mathrm{X}$ syndrome is related to FMR1 protein expression. Human Brain Mapping, 16 (4), 206-218. doi:10.1002/hbm.10048

Roberts, J. E., Hennon, E. A., Price, J. R., Dear, E., Anderson, K., Vandergrift, N. A. y Abbeduto, L. (2007). Expressive Language during Conversational Speech in Boys with Fragile X Syndrome. American Journal on Mental Retardation, 112 (1), 1-17. doi:10.1352/0895-8017(2007)112[1:ELDCSI]2.0.CO;2

Roberts, J., Long, S. H., Malkin, Ch., Barnes, E., Skinner, M., Hennon, E. A. y Anderson, K. (2005). A Comparison of Phonological Skills of Boys with Fragile X Syndrome and Down Syndrome. Journal of Speech, Language and Hearing Research, 48, 980-995. doi:10.1044/1092-4388(2005/067)

Roberts, J., Martin, G. E., Moskowitz, L., Harris, A. A., Foreman, J. y Nelson, L. (2007). Discourse Skills of Boys with Fragile X Syndrome in Comparison to Boys with Down Syndrome. Journal of Speech, Language and Hearing Research, 50, 475-492. doi:10.1044/1092$4388(2007 / 033)$

Rondal, J. A. (2007). Teoría de la mente y lenguaje. Revista de Logopedia, Foniatría y Audiología, 27 (2), 51-55. doi:10.1016/S0214-4603(07)70073-6

Ruggieri, V. L. y Arberas, C. L. (2003). Fenotipos conductuales. Patrones neuropsicológicos biológicamente determinados. Revista de Neurología, 37 (3), 239-253.

Sullivan, K., Hatton, D., Hammer, J., Sideris, J., Hooper, S., Ornstein, P. y Bailey, D. (2006). ADHD symptoms in children with FXS. American Journal of Medical Genetics, Part A, 140A (21), 2275-2288. doi:10.1002/ajmg.a.31388

Sullivan, K., Hatton, D. D., Hammer, J., Sideris, J., Hooper, S., Ornstein, P. A. y Bailey, D. B. (2007). Sustained attention and response inhibition in boys with fragile $\mathrm{X}$ syndrome: Measures of continuous performance. American Journal of Medical Genetics Part B: Neuropsychiatric Genetics, 144B (4), 517-532. doi:10.1002/ajmg.b.30504

Visootsak, J., Warren, S. T., Anido, A. y Graham, J. M. (2005). Fragile X Syndrome: An Update and Review for the Primary Pediatrician. Clinical Pediatrics, 44, 371-381. doi:10.1177/000992280504400501

(C) Ediciones Universidad de Salamanca

Siglo Cero, vol. 46 (4), n. ${ }^{\circ}$ 256, 2015, octubre-diciembre, pp. 25-44 